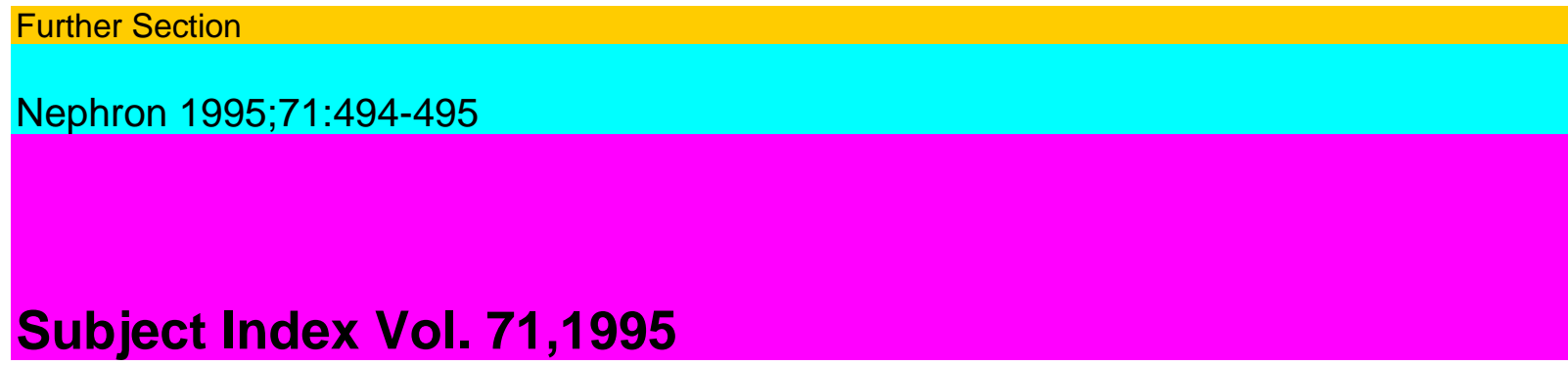

\title{
Acetate 138
}

Acute renal failure 59, 203, 309, 373, 428,

454 Adenosine 184 Adriamycin nephropathy 87 Aldosterone 44 Alphacalcidol 254 Amiloride 87 Amino acid sequence 160 Ammonia 433

Antigen-bound immunoglobulin 448 Antioxidant enzymes 176 Antiphospholipid antibodies 35 Antirejection therapy, high-dose steroids 40 Anti-Thy-1.1 antibody 79 Atria contractility 190 Atrial natriuretic peptide 44 Azotemia, prerenal 153

Bacterial killing 133 Bicarbonate 138 Blood pressure 44 Bone 218 Buffers 138

CaC03 350 Calcitonin 428 Calcitriol 218,254

- $\quad$ pulse therapy 350

Captopril 208

Carbamylated hemoglobin 153 Cell division 433 Chlorambucil 373 Cholangiocarcinoma 354

Cholestasis 354 Chondroitin sulfate 197 Chronic active hepatitis 156,213

ambulatory peritoneal dialysis 297

renal failure 160,190 Cirrhosis 354 Coagulation 149 Collagen 197

Continuous ambulatory peritoneal dialysis

16,23,133,314 Crescentic glomerulonephritis 303 Critical illness 59

Cyclic guanosine monophosphate 44 Cyclophosphamide 321, 373 Cyclosporine 180,373 Cystine 328, 407 Cystinuria 328,407,416 Cytokines 309

Ddimers 149 Deflazacort 373 Diabetes mellitus 54 Dialysate flow rate 401 -, saving 401 Dialysis 254,350

- $\quad$ optimization 401

Dietary sodium 407

Divalent ions 350

DNA synthesis 433

Dobutamine 168

Dopamine 168

Doppler ultrasound 168

Electrophoresis 160 Endothelin 44,291 End-stage renal failure 133 Enzyme-linked

immunosorbent assay 75 Erythropoietin 190,395 Exertional heat stroke 428 Extracellular matrix proteins, immunohisto-chemical quantitation 79

Femoral artery 95 Fibronectin 197 Folic acid deficiency 395 Fragment $1+2149$ Free radicals 176

Gentamicin 203

Glomerular basement membrane 442

filtration rate 10

hypertrophy 208

injury 87 Glomerulonephritis 454 -, rat model 79 Glomerulosclerosis 208 Glycerol-induced acute renal failure 184 
Hemoconcentration 65 Hemodialysis 59, 65,138, 156, 160,218 Hemodilution 65 Hemofíltration 59 Hemolytic-uremic syndrome 54, 309 Hemorrhagic fever, renal syndrome 419 Heparan sulfate 197 Heparin 95, 149 Hepatitis 386

C, chronic active 156

- virus 156,213,386 Heymann nephritis, active $448 \mathrm{HgC}^{1 / 8} /$-induced acute renal failure 184 Highsalt diet 87

HLA-DR phenotype 180 Hyperbilirubinemia 354 Hyperglycemia 54 Hyperparathyroidism 254, 350 Hypersplenism 343 Hypertension, renovascular 291 Hypertrophy, glomerular 87

Hypoalbuminemia 314 Hypouricemia 354

IgA nephropathy 75

Immunodetectable porphobilinogen deami-

nase 297 Immunoglobulin 454 Immunosuppression 176 Infrared spectroscopy 160 In situ

hybridisation 303 Insulin-like growth factor 116 Intact parathyroid hormone 428 Intensive care

59 Interferon 156,213 Interleukin(s) 309,442

- receptor 303

Intermittent hemodialysis 297

Intraperitoneally administered agents 23

Kidney 386

- $\quad$ section 79

Laminin 197

Late acute transplant rejection 40

Levamisole 373

Lipids 65

Lithium 44

Low-molecular-weight heparin 149

Low-protein diet 208

Lupus anticoagulant 35

nephritis 321

nephropathy 35

Macrocytic anemia 395

Matrix 197

Mean corpuscular volume 395

2-Mercaptopropionylglycine 328, 407

Mesangial cells 197

Methylprednisolone 373

Middle molecules 160

Milan normotensive rats 208

$\mathrm{Na}+/ \mathrm{H}+$ exchanger inhibitor 87 Nephrotic syndrome 44, 95, 149, 373 Nephrotoxicity 203

Neutropenia 454

494

Nitric oxide 203 Nitrogen mustard $373^{\wedge}$-Nitrophenylphosphatase activity 190 Noradrenaline 184 Normal rat kidney cells 433 Nutrition 16

OKT3 180

Orally administered calcium 254

Osteocalcin 428

Ouabain 190 
Oxidative injury 343

Parathyroid hormone 218,284 Pelvic-ureteral obstruction 143 Peritoneal albumin loss 314 dialysis 29,59

mesothelial cell growth 23

solute transport 314 Peritonitis 373 Phagocytosis 133 Phosphate 10

binder 254

Plaque reduction neutralization test 419 Polymeric immunoglobulin receptor 75

Polymorphonuclear leukocytes 176

- neutrophils 133

Porphobilinogen deaminase 297

Porphyrins 297

Potassium 10 Prednisolone 373

Primary malignant hypertension 29 Prolactin 284 Proteins 65

Recombinant human erythropoietin 297 ,

343 Red cell deformability 343 Reduced glutathione 343 Renal anemia 343,395

biopsy 373

bone disease 254

failure, acute, chronic 29

function 44

hemodynamics 168

plasma flow 10

proximal tubular antigen 448

transplantation 40, 180, 213, 373, 386

vasoconstriction 184 Respiratory burst 176 Rhabdomyolysis 428

Scavengers 176 Sclerosis, glomerular 87 Secondary hyperparathyroidism 284 Secretory

component 75

- $\quad$ immunoglobulins 75

Seoul virus 419

Sleep apnea 138

Sodium 10

-, dietary 416

Soluble tumor necrosis factor receptors 309

35Sulfate uptake 442 Superoxide anion 133 Suspected urinary tract obstruction, ultrasound diagnosis 143 Systemic lupus erythematosus 321

Thrombocytopenia 454 Thrombosis 95 Thyroid hormones 284 Thyrotropin 284 Tiopronin 328,407

Tiopronin-cysteine-mixed disulfide 328 Tiopronin-cystine-mixed disulfide 407 Tumor necrosis factor alpha 180,309

Urea 433 Uremia 176,254 Urinary calculi 416 Urodynamic tests 143

Valine hydantoin 153 Viral infection 386 Virus disease 454 Vitamin D 254 - - metabolites 428

Warfarin 95 Weight gain 65

495 